\title{
PSICOLOGÍA
}

\section{Riesgo suicida en mujeres en situación de privación de libertad}

\author{
Valentina Vargas ${ }^{1}$, Cristina Arrom Suhurt ${ }^{1,2}$, Miguel Cuellar ${ }^{1}$, \\ María del Pilar Fresco ${ }^{1}$, Carmen Marina Arrom Suhurt², \\ María Auxiliadora Arrom²
}

\begin{abstract}
Resumen
Introducción: El suicidio es un problema grave de salud pública con factores que aumentan el riesgo. Esta conducta abarca un amplio espectro, desde la ideación suicida, preparación de un plan, obtención de los medios para llevarlo a cabo, hasta la posible consumación del acto. En la actualidad el suicidio aqueja a gran parte de la población mundial, aproximadamente un millón de personas mueren cada año en el mundo por suicidio. En promedio se suicidan a diario tres mil personas y, por cada una, veinte lo intentan. En todos los países el factor de encarcelamiento multiplica la tasa de suicidio señala un estudio realizado en Cataluña, constituyendo una causa común de muerte en prisión. Esto se debe a que la privación de la libertad impacta de manera significativa en las personas que se encuentran en esta condición. Influye en el proyecto de vida del interno, afecta su salud mental, como estigma, cambio de modus vivendi, lo separa de familiares y otras fuentes de apoyo social. Se suman a estos factores las condiciones de la mayoría de los sistemas carcelarios deficientes, que violan los derechos humanos de los presidiarios por el hacinamiento, atención deficitaria a la salud, personal de guardia inadecuado, mezcla de la población carcelaria, entre otras En Paraguay ha habido un aumento en la tasa de suicidios en los últimos 5 años, siendo de 5,64 por cada 100.000 habitantes en el 2009 y de 6,81 en el 2014. La edad es otro factor de riesgo asociado, señala otro estudio, donde a nivel país se dieron más frecuentemente los suicidios en el rango de entre 18 a 29 años de edad.
\end{abstract}

Objetivo: Determinar el riesgo de suicidio que tenían mujeres privadas de libertad en una Correccional.

Material y Método: Estudio descriptivo, comparativo y correlacional de corte transversal con componente analítico. Muestra no probabilística, por conveniencia, de 108 mujeres de una Correccional de Mujeres en Paraguay. Se utilizó un cuestionario socio demográfico y la Escala de Suicidio de Plutchik previa firma de consentimiento informado que explicó los objetivos del estudio

1. Cátedra de Psiquiatría, Facultad de Ciencias Médicas, Universidad Nacional de Asunción, Paraguay.

2. Centro para el Desarrollo de la Investigación Científica (CEDIC), Paraguay.

E-mail: crisarron@gmail.com

DOI: 10.26885/rcei.foro.2017.124 


\section{Riesgo suicida en mujeres en situación de privación de libertad. Vargas et al.}

y la participación voluntaria y anónima. Paquete estadístico SPSS versión 15.0 para el análisis de la información.

Resultados: 36,1\% (39) de la muestra de estudio tenía entre 32 y 43 años; 34\% (37) entre 20 y 31 y $28,7 \%$ (31) más de 43 años; 0,9\% (1) no respondió. El 38,9\% (42) tenía primaria (completa e incompleta); 44,5\% (48) secundaria (completa e incompleta); $12 \%$ (6) terciaria (completa e incompleta) y 4,6\% (5) no respondió. Se encontró un 36,1\% de riesgo suicida en las mujeres encuestadas, en tanto un $63,9 \%$ (69) sin riesgo. El 25,9\% (28) tenía riesgo leve; 6,5\% (7) riesgo moderado y $3,7 \%$ (4) riesgo grave. Cifra de riesgo encontrada en el estudio fue mucho mayor a la población general y a cifras arrojadas por otros estudios similares en centros penitenciarios en América Latina. Este porcentaje puso en evidencia que en la institución un porcentaje importante de mujeres privadas de libertad se encontraba en situaciones graves de trastornos de salud mental sin que las mismas hayan sido detectadas institucionalmente como oportunidad para intervenir y evitar muertes.

Conclusiones: La reclusión representa una gran dificultad para la persona que la experimenta, ya que no solo representa una pérdida de libertad, confianza en sí mismo, credibilidad y respeto frente a los demás, sino también, en muchos casos, la pérdida del apoyo familiar y social. Esta condición promueve el desarrollo de desesperanza y depresión que aumentan el riesgo que tienen mujeres en condiciones de reclusión, de fantasear con la muerte como alternativa de escape a una situación a la que no pueden adaptarse. Limitaciones que presentó este trabajo: en primer lugar, pruebas psicológicas utilizadas no han sido validadas con muestras paraguayas, a pesar de su amplio uso en el ámbito clínico y los datos de validez y confiabilidad que tienen internacionalmente. No obstante, la coherencia teórica de estos resultados confirma que estos instrumentos son válidos en constructo como lo demuestra la consistencia interna arrojada en esta investigación respecto a la Escala de Riesgo Suicida de Plutchik y el Inventario de Depresión.

Se requiere la presencia de programas de salud mental en población carcelaria que involucren al personal penitenciario en general así como la tarea de médicos psiquiatras y psicólogos para disminuir el impacto de ciertos trastornos mentales y ayudar a las personas recluidas a hacer frente al estrés psicológico y emocional de su condición.

Palabras clave: riesgo suicida, mujeres, privación de libertad.

\section{Referencias}

Adorno, S. C. \& Pagliaro, N. R. (2012). Situación actual del suicidio y tentativa de suicidio en el Paraguay. An. Fac. Cienc. Méd., 45( 2 ), 25-38.

Bedoya, A., Martínez-Carpio, P., Humet, V., Leal, M. \& Lleopart, N. (2015). Incidence of suicide in Catalán prisons: a descriptive and comparative study. Revista Española De Sanidad Penitenciaria, 11(2), 37-41. 
De la Rubia J. Validación de un formato simplificado del Inventario de Depresión de Beck (BDI-2). = Validation of a simplified form of Beck Depression Inventory (BDI-2). Psicología Iberoamericana, 21(1), 42-52.

Mojica, C., Sáenz, D. \& Rey-Anacona, C. (2009). Riesgo suicida, desesperanza y depresión en internos de un establecimiento carcelario colombiano. Revista Colombiana De Psiquiatría, 38(4), 681-692.

OMS. (2016). Suicidio. Recuperado de http://www.who.int/mediacentre/ factsheets/fs398/es/

Rubio, G., Montero, I., Jáuregui, J., Villanueva, R., Casado, M. A., Marín, J. J., et al. (1998). Validación de la escala de riesgo suicida de Plutchik en población española. Arch Neurobiol., 61(2),143-52.

Rubio, L. A. (2014). Riesgo suicida en población carcelaria del Tolima, Colombia. Revista de la Facultad de Medicina, 62(1), 33. Recuperado de http://www.revistas.unal.edu.co/index.php/revfacmed/article/ view/43672/47372 